\title{
CloudSimDisk: Energy-Aware Storage Simulation in CloudSim
}

\author{
Baptiste Louis, Karan Mitra, Saguna Saguna and Christer Åhlund \\ Department of Computer Science, Electrical and Space Engineering \\ Luleå University of Technology, Skellefteå, Sweden \\ Emails: baptiste_louis@live.fr, \{karan.mitra, saguna.saguna, christer.ahlund $\} @ 1 t u . s e$
}

\begin{abstract}
The cloud computing paradigm is continually evolving, and with it, the size and the complexity of its infrastructure. Assessing the performance of a cloud environment is an essential but an arduous task. Further, the energy consumed by data centers is steadily increasing and major components such as the storage systems need to be more energy efficient. Cloud simulation tools have proved quite useful to study these issues. However, these simulation tools lack mechanisms to study energy efficient storage in cloud systems. This paper contributes in the area of cloud computing by extending the widely used cloud simulator CloudSim. In this paper, we propose CloudSimDisk, a scalable module for modeling and simulation of energy-aware storage in cloud systems. We show how CloudSimDisk can be used to simulate energy-aware storage, and can be extended to study new algorithms for energy-awareness in cloud systems. Our simulation results proved to be in accordance with the analytical models that were developed to model energy consumption of hard disk drives in cloud systems. The source code of CloudSimDisk is also made available for the research community for further testing and development.
\end{abstract}

Index Terms-Cloud Computing, CloudSim, Energy Efficiency, Modeling, Simulation, Storage

\section{INTRODUCTION}

The "Digital Revolution" of the past decades has marked the beginning of the "Information Age", characterized by the development of Information and Communication Technologies (ICTs) and transformed the way in which we communicate, produce data. In parallel, the issues around global warming are unprecedented and indicate a significant human disruption since the beginning of the industrial revolution. The SMART 2020 report published by GeSI [1] states that "ICT is a key sector in the fight against climate change". Additionally, in the GeSI report, data centres have been identified as the "fastest-growing contributor to the ICT sector's carbon footprint". ICTs are generating a large volumes of data which is constantly shared, copied and exchanged between multiple entities. Cloud computing reveals to be a powerful tool to deal with the challenges associated with Big Data issues. However, this solution is causing the multiplication of large-scale data centers consuming tremendous amount of energy [2], and with a degree of complexity making them difficult and costly to monitor, expand, and optimize, both in terms of performance and energy.

Cloud simulation tools empower researchers to study and analyze all layers (Infrastructure-as-a-Service, Platform-as-aService, and Software-as-a-Service) of the cloud environment in a cost-efficient, stable and scalable manner. CloudSim [3] is a popular discrete-event simulator tool for cloud environment, allowing modeling and simulation of cloud computing infrastructures and services. While storage system is a major part of a cloud environment, CloudSim offers insufficient modeling and simulation capabilities to study energy-aware storage in cloud systems.

In this paper, we propose, develop and validate CloudSimDisk, a scalable module enabling energy-aware storage simulations in CloudSim. It's implementation is based on analytical models that were tested against hard disk drive manufacturer specifications. Further, as CloudSimDisk was developed to be flexible, it can easily be extended by the researchers and cloud practitioners to test and validate their algorithms and systems for energy-aware storage in cloud systems.

This paper is organized as follows: Section II provides the background and related work. Section III presents CloudSimDisk, a module for energy-aware storage simulations in CloudSim. Section IV presents the results analysis. Finally, Section $\mathrm{V}$ presents the conclusion and future work.

\section{BACKGROUND AND RELATED WORK}

In this section, we begin by presenting a brief but essential background on the CloudSim simulator [3]. Next, CloudSim extensions, closely related to our work, are discussed. CloudSim is an event-based simulator: events can be generated dynamically, and they are executed chronologically. One major component of CloudSim is the datacenter entity, which aims to model a real data center. It houses a list of Hosts (physical servers machines) with defined hardware specifications (RAM, Bandwidth, Capacity, CPUs), on which several Virtual Machines (VMs) can be executed. Additionally, the datacenter entity contains one storage component, defined as a list of Hard Disk Drive (HDD) element. However, CloudSim does not provide any example of interaction with this component, and a close analysis of it's implementation reveals a clear lack in storage modeling.

To the best of our knowledge, only three projects aim to tackle the storage component in CloudSim. For instance, Sturm, Jrad, and Streit [4] presents an extension for Storageas-a-Service (STaaS) modeling in CloudSim. Grozev and Buyya [5] proposes a hard disk drive (HDD) processing element representing the $\mathrm{I} / \mathrm{O}$ capacity of a storage disk. 
Finally, long and Zhao [6] adds file striping and data replica features to the CloudSim simulator. In contrast with the discussed extensions, we propose ad develop a scalable and a flexible module, that allows energy-aware storage simulation in CloudSim, including HDD device models, HDD power models, and energy-aware data center persistent storage.

\section{CloudSimDisk: Energy-Aware Storage Simulation In CloudSim}

This section presents the CloudSimDisk module enabling modeling and simulation of energy-aware storage in the CloudSim simulator.

\section{A. Design}

CloudSimDisk extends CloudSim Toolkit v3.0.3 and follows the same design pattern (see Figure 1), so users will be able to use new implemented features, or extend them, with a minimum learning curve. In addition, the module provides modeling of HDD components and their related power models.

1) HDD models: The main characteristics affecting the overall HDD performance are the mechanical components, combination of the read/write head transversal movement, and the platter rotational movement. Additionally, the internal data transfer rate, often called sustained rate, is usually considered to be a bottleneck for the overall data transfer rate of an HDD. Thus, the cloudsimdisk.models.hdd package has been created that contains classes modeling HDD storage components. Each model implements one method, namely getCharacteristic(int key). In this method, the parameter key is used in a "Switchcase" statement which returns the value of a specific characteristic of this HDD. Table I lists the characteristics implemented in CloudSimDisk. Furthermore, all the classes modeling an HDD extend a common abstract class, that provides a consistent and semantically understandable interface to access a specific characteristic of an HDD object.

2) HDD power models: CloudSim allows simulation of energy-aware servers, both the physical host and the virtual machine. However, it does not provide energy-awareness for the data center persistent storage and its HDD components. Therefore, CloudSimDisk module provides a power model for each HDD model implemented. For that purpose, the package cloudsimdisk.power.models.hdd has been created in accordance with the power package in CloudSim. Within this package, the abstract class PowerModelHdd.java implements semantically understandable getters to retrieve the power consumption of an HDD in a particular operating mode, either Active mode (the disk is reading or writing) or Idle mode (the disk is spinning but there is no activity on it).

\section{B. Components}

1) Data Cloudlet: In CloudSim, a Cloudlet models the cloud-based application services (i.e., a job or a task). In CloudSimDisk, the Cloudlet model is reused to model an incoming request to the data center persistent storage. The initial Cloudlet properties are not used in CloudSimDisk. Instead, two new parameters has been defined:

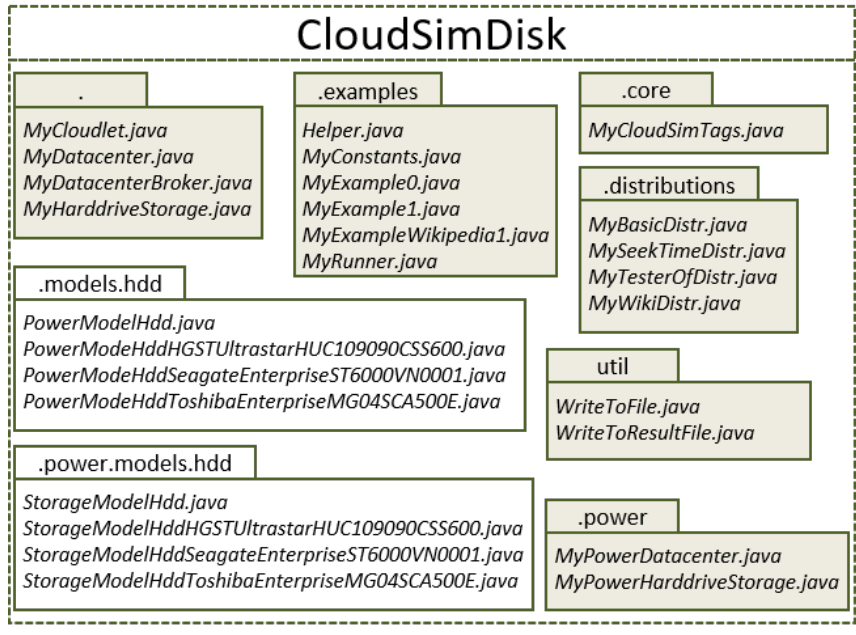

Package extended from CloudSim New CloudSimDisk package

Fig. 1: CloudSimDisk package diagram.

TABLE I: CloudSimDisk HDD model characteristics.

\begin{tabular}{|c|l|l|}
\hline KEY & CHARACTERISTICS & DESCRIPTION \\
\hline 0 & Manufacturer Name & $\begin{array}{l}\text { The name of the Manufacturer } \\
\text { (Ex: Seagate Technology, Toshiba, } \\
\text { Western Digital). }\end{array}$ \\
\hline 1 & Model Number & $\begin{array}{l}\text { The unique manufacturer reference } \\
\text { (Eg., ST4000DM000). }\end{array}$ \\
\hline 3 & Capacity & $\begin{array}{l}\text { The capacity of the HDD in } \\
\text { megabyte (MB). }\end{array}$ \\
\hline 4 & Aatency & $\begin{array}{l}\text { The average rotation latency of the } \\
\text { disk which is defined as half the } \\
\text { amount of time it takes for the disk to } \\
\text { make one full revolution, in second } \\
\text { (s), directly dependent on the disk ro- } \\
\text { tation speed in Rotation Per Minute } \\
\text { (RPM). }\end{array}$ \\
\hline 5 & $\begin{array}{l}\text { Maximum } \\
\text { Data Transfer Rate } \\
\text { which is defined as the average time } \\
\text { needed to move the read/write head } \\
\text { from track x to track y, also corre- } \\
\text { sponding to one-third of the longest } \\
\text { possible seek time, moving from } \\
\text { the outermost track to the innermost } \\
\text { track, assuming an uniform distribu- } \\
\text { tion of requests. }\end{array}$ \\
\hline $\begin{array}{l}\text { The maximum internal data transfer } \\
\text { rate which is defined as the rate at } \\
\text { which data is transferred physically } \\
\text { from the disk to the internal buffer, } \\
\text { also called Sustained Data Rate or } \\
\text { Sustained Transfer Rate. }\end{array}$ \\
\hline
\end{tabular}

- requiredFiles: a list of filenames that need to be accessed by the Cloudlet. These files have to be on the persistent storage of the data center before the Cloudlet is processed.

- dataFiles: a list of files that need to be stored by the Cloudlet. These new files will be added to the persistent storage of the data center during the Cloudlet processing. 
File sizes and file names information for both requiredFiles and dataFiles are retrieved from raw text files. Note that requiredFiles has already been implemented in CloudSim but it has been called fileList. However, this list is not a list of File objects, but a list of String objects, and the new parameter dataFiles, implemented in CloudSimDisk, is a list of File objects. Thus, to make things more clear, the fileList parameter has not been reused by CloudSimDisk.

2) Data center: In CloudSim, one parameter of the data center entity is a list of storage elements. This list models the data center persistent storage. Unfortunately, CloudSim does not provide any information on how to interact with this component. Thus, an extension of the CloudSim data center model has been realized in CloudSimDisk. All necessary algorithms to process requiredFiles and dataFiles of a Cloudlet have been implemented. Additionally, the addFile() method, responsible for adding a file to the persistent storage, has been improved to be more flexible.

3) Broker: The broker entity implemented in CloudSimDisk is responsible to send Cloudlets one by one to the datacenter entity. Each Cloudlet is scheduled at a specific time, depending on the distribution defined in the simulation settings. CloudSim provides common distribution generators such as uniform, exponential and logarithmic normal distributions. Additionally, CloudSimDisk allows users to read arrival times from a raw text file, which can be manually created files or traces from real-world cases.

\section{Randomized Characteristics}

In the real world, most of the HDD characteristics vary over time, affected by numerous internal factors (e.g., physical location of data and data fragmentation) and external factors (e.g., rack vibrations, and temperature and humidity of the operating environment.). In CloudSimDisk, two key HDD characteristics are randomized: rotation latency and seek time. The rotation latency is generated from a uniform distribution, varying between 0 and 2 times the average rotation latency of the HDD. The seek time is generated from a distribution which returns a series of random numbers between 0 and 3 times the average seek time, and with an average of $\frac{1}{3}$ of the average seek time of the HDD [7].

\section{Module Scalability}

The CloudSimDisk implementation follows the CloudSim design and meets several quality attributes of software engineering concepts. As a consequence, the proposed module is easily understandable and scalable. Both the HDD model and the HDD power model are implemented with extensibility in mind, allowing the declaration of additional device characteristics, and power data, for future improvement. Similarly, CloudSimDisk allow the user to test their own algorithms for disk array management, seek time distribution, or Cloudlet arrival rate.

\section{E. Cloudlet Processing}

CloudSimDisk uses the core simulation engine of CloudSim. Hence, the simulation process is identical in both

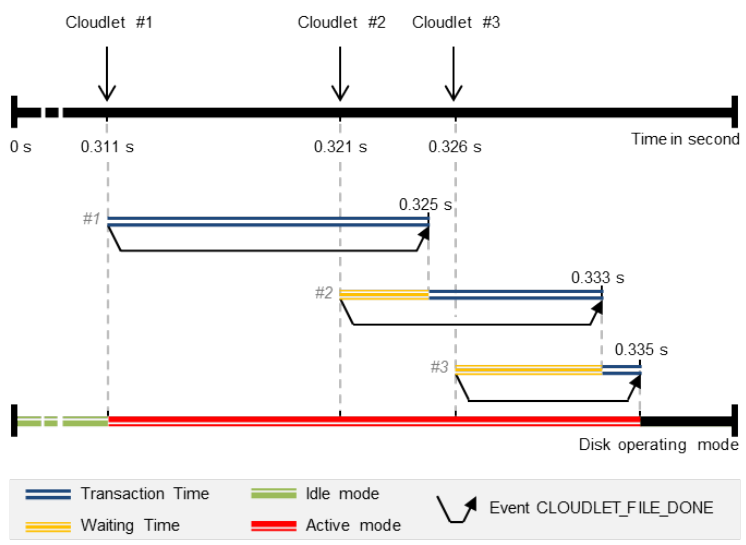

Fig. 2: Sequential processing of incoming Cloudlets.

cases. However, CloudSimDisk processes Cloudlets in a different manner, since the CloudSimDisk module interacts with the data center storage (see Figure 2). The arrival times of Cloudlets depend on the user configuration. Chronologically, each Cloudlet is processed: dataFiles are added to the data center storage and requiredFiles are retrieved. These interactions with an HDD is called a transaction. The duration of the transaction is the sum of the seek time, the rotation latency and the transfer time (see formula (1)). If the target HDD is already handling one or more other transactions, a waitingTime is computed (see formula (2)). The activeEndAt time is the time at which the HDD will have handled all its transactions.

$$
\begin{gathered}
t_{\text {transactionTime }}=t_{\text {seekTime }}+t_{\text {rotLat }}+t_{\text {transfTime }} \\
t_{\text {waitingTime }}=t_{\text {activeEndAt }}-t_{\text {currentTime }}
\end{gathered}
$$

\section{F. Energy-Awareness}

Concerning energy-awareness, each HDD model contains an HDD power model. A disk is in active mode when handling some transactions, and in idle mode otherwise (see Figure 2). The total energy consumed by an HDD is computed according to the time spent in each mode. Later, the intervals during which the HDD was in idle mode can be retrieved. The durations of these intervals can be used to determine energy saving strategies, e.g., by shutting down some underused HDDs. At the data center level, the total energy consumed by the persistent storage during a simulation is computed. The energy considered is the sum of the energy consumed to perform each read/write request on the persistent storage plus the energy consumed during idle mode intervals.

\section{G. Analytical Model}

The final result of a CloudSimDisk simulation is the total energy consumed by the data center persistent storage, during a simulation. The energy consumed by the persistent storage is noted $E_{\text {persistentStorage. }}$. It is obtained by the sum of all $E_{\text {hdd_i }}$, the energy consumed by the $i$-th HDD out of $n$ in the persistent storage (see formula (3)). 


$$
E_{\text {persistentStorage }}=\sum_{i=1}^{n} E_{h d d_{-} i}
$$

Then, the energy consumed by the $i$-th HDD $E_{h d d_{-} i}$ is the sum of the energy consumed by this HDD in Idle mode $E_{h d d_{-} i \text {, idle }}$ and in Active mode $E_{h d d_{-} \text {, active }}$ (see formula (4)).

$$
E_{h d d_{\_} i}=E_{h d d_{-} i, \text { idle }}+E_{h d d_{-} i, \text { active }}
$$

The energy consumed in Idle mode for a specific HDD

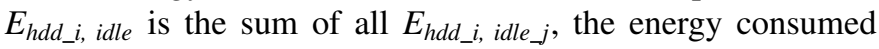
by the $i$-th HDD during the $j$-th idle interval out of $m$ (see formula (5)).

$$
E_{h d d_{-} i, i d l e}=\sum_{j=1}^{m} E_{h d d \_i, i d l e \_j}
$$

The energy consumed in Active mode for a specific HDD $E_{\text {hdd_i, active }}$ is the sum of all $E_{\text {hdd_i, active_j, the energy consumed }}$ by the $i$-th HDD during the $j$-th transaction out of $m$ (see formula (6)).

$$
E_{h d d \_i, \text { active }}=\sum_{j=1}^{m} E_{h d d \_i, a c t i v e \_j}
$$

The energy consumed by a specific HDD during a specific Idle interval $E_{h d d{ }_{-}, \text {idle } j}$ is the interval duration $t_{\text {idle } j}$ multiplied by the power required by the HDD in Idle mode $P_{h d d \_ \text {, idle }}$ (see formula (7)).

$$
E_{h d d_{-} i, i d l e_{-} j}=t_{i d l e_{-} j} \times P_{h d d_{-} i, i d l e}
$$

The energy consumed by a specific HDD during a specific transaction $E_{\text {hdd_i, active } j \text { is }}$ is the transaction duration $t_{\text {hdd_i, transaction_j }}$ multiplied by the power required by the HDD

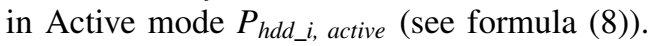

$$
E_{h d d_{\_} i, \text { active }_{\_} j}=t_{h d d_{\_} i, \text { transaction } \_j_{j}} \times P_{h d d_{\_} i, \text { active }}
$$

\section{Simulation Results}

This section contains a series of results obtained with the CloudSimDisk module.

\section{A. Comparison With Analytical Model}

A transaction on the persistent storage consists of adding/retrieving a file to/from an HDD. A file has a name and a size. While the name is used to identify each file, the size has an impact on the energy consumed by the storage. Indeed, in a simple manner, the transfer time can be expressed as the data transfer rate of the target HDD multiplied by the size of the file to process. Figure 3 shows the energy consumption per transaction depending upon the size of the file processed. The scenario consists of adding 100 files with the same size to the persistent storage. The HDD model used is a HGST Western Digital 900GB, with an average seek time

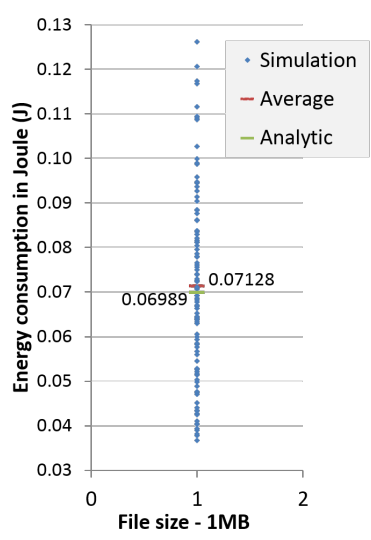

(a)

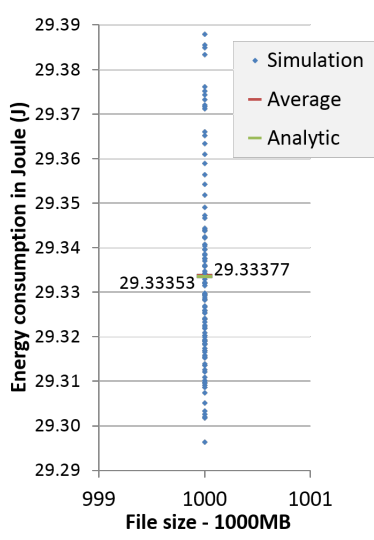

(b)
Fig. 3: Energy consumed per transaction $E_{\text {transaction }}$ with file sizes of (a) $1 \mathrm{MB}$, (b) $1000 \mathrm{MB}$.

of 0.003 second, an average rotation latency of 0.004 second and a maximum internal data transfer rate of $198.0 \mathrm{MB} / \mathrm{s}$ (ref: HUC109090CSS600). It consumes around $3.0 \mathrm{~W}$ in idle mode and $5.8 \mathrm{~W}$ in active mode. The scenario has been repeated with different file sizes of 1, 10, 100 and 1000 megabytes.

To verify the validity of the simulations, analytical results were calculated according to formula (9). The energy consumed by the transaction is noted as $E_{\text {transaction. }} . f_{\text {size }}$ is the size of the file concerned by the transaction.

$$
\begin{aligned}
& E_{\text {transaction }}=\left[t_{\text {avgSeekTime }}+t_{\text {avgRotLat }}\right. \\
& \left.+\left(t_{\text {max IntDataTransfRate }} \times f_{\text {size }}\right)\right] \times P_{\text {active }}
\end{aligned}
$$

The results are in accordance with the analytical values. The tiny difference is explained by the randomness of the seek time and the rotation latency (see Figure 3). Nevertheless, the means obtained for each simulation are very close to the analytical results. This shows that the seek time and the rotation latency vary around the average values defined in the HDD model used as input parameter of the simulation.

\section{B. Disk Array Management}

When Cloudlets are processing, new files are eventually stored in the persistent storage, which is composed of one or more HDDs. To define a target HDD for each transaction, CloudSim implements a FIRST-FOUND algorithm: it scans the list of available HDD and add the file on the first one which have enough free space for the file. CloudSimDisk additionally implements a ROUND-ROBIN algorithm: files are added sequentially to each disk of the persistent storage. When the algorithm reaches the end of the disk array, it restarts from the first disk. If one disk is full, the algorithm tries the next one. Figure 4 presents the results of three similar scenarios, using Round-Robin, with a variable persistent storage size of 1 HDD, 2 HDDs and 3 HDDs. When the persistent storage is composed of only $1 \mathrm{HDD}$, the coming requests tends to accumulate in the disk queue leading to poor performances. 


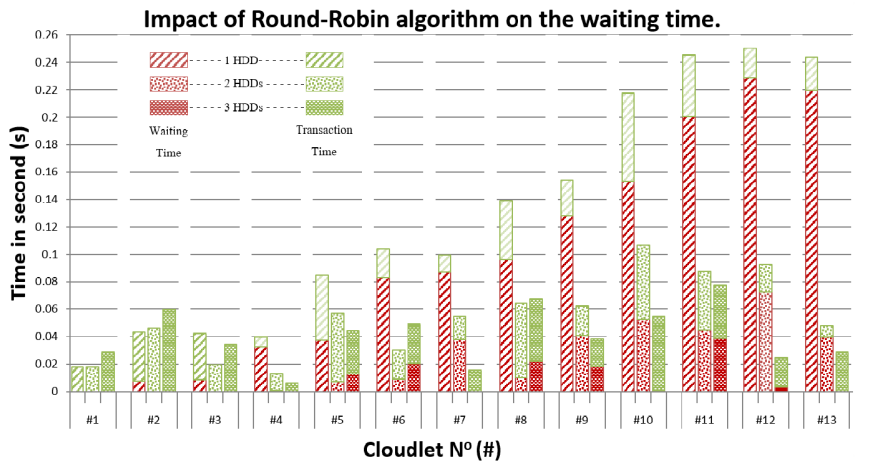

Fig. 4: CloudSimDisk experiments with 1 HDD, 2 HDDs and 3 HDDs in the persistent storage using Round-Robin algorithm.

When the persistent storage is composed of 2 HDDs, the ROUND-ROBIN algorithm balances the incoming requests on both drives, reducing significantly their "waiting time" and improving the overall performances of the persistent storage. When the persistent storage is composed of 3 HDDs, the load balancing is even more effective. Most of the Cloudlets had a null "waiting time". To summarize, CloudSimDisk has proved the performances of the Round Robin algorithm for disk array management. Later, new algorithms can be implemented and tested.

\section{Case Study: Wikipedia Trace}

To test the CloudSimDisk module under realistic workload, we used a Wikipedia trace from September 2007. The request arrival rate was approximately 3000 requests per second. We assumed that each request adds one file to the persistent storage. The file sizes were variable and were distributed between $1 \mathrm{MB}$ to $10 \mathrm{MB}$. The persistent storage was composed of 1 HDD. The model of the HDD was considered to be a HGST Ultrastar (Ref: HUC109090CSS600). It consumed around $3.0 \mathrm{~W}$ in Idle mode and $5.8 \mathrm{~W}$ in Active mode. Its average seek time was considered to be 0.003 second. Its average rotation latency was 0.004 second, and the maximum internal data transfer rate was $198 \mathrm{MB} / \mathrm{s}$. [7] We considered 5000 requests, i.e., around 1.7 second of Wikipedia trace. The total energy consumed by the persistent storage was 1010.927 Joules. The transfer rate (see Figure 5), the average seek time and the average rotation latency obtained by simulations were in accordance with the HGST Ultrastar HDD characteristics. This result further shows the efficacy of CloudSimDisk for modeling accurate energy-consumption of HDDs in the cloud datacenters.

\section{CONCLUSION AND FUTURE WORK}

In this paper, we proposed CloudSimDisk, a module for energy-aware storage simulation in the widely used CloudSim simulator. The implementation includes HDD models, HDD power models, disk array management algorithms and energyaware data center storage. The input workload used for the simulations can be read from a file, that empower users to

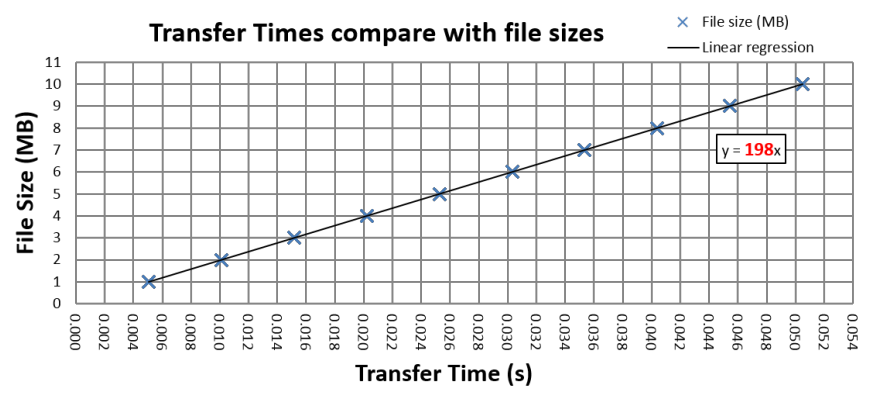

Fig. 5: Transfer times and file sizes.

test their own traces, and allows others to repeat similar experiments. The experiments realized with the proposed module proved the exactness of simulation results compared with the described analytical model. Moreover, they validate the implementation of Round-Robin algorithm for disk array management and the seek time and rotation latency distributions. Further, the scalability of the module allows future implementations of more complex algorithms.

As future work, simulation results need to be compared with real-world measurements. The transfer time considered in CloudSimDisk should be improved and, disk caching and disk fragmentation should be considered.

Software availability: CloudSimDisk source code has been released as open source software and can be downloaded from: https://github.com/Udacity2048/CloudSimDisk. It includes several examples and various tutorials.

\section{ACKNOWLEDGMENT}

The authors would like to thank the PERCCOM program and the Erasmus+ program for providing funding for this thesis work. They are also grateful to Rodrigo N. Calheiros for his guidance during the development of CloudSimDisk reported in this paper.

\section{REFERENCES}

[1] M. Webb et al., "Smart 2020: Enabling the low carbon economy in the information age," The Climate Group. London, vol. 1, no. 1, pp. 1-1, 2008.

[2] T. Mastelic, A. Oleksiak, H. Claussen, I. Brandic, J.-M. Pierson, and A. V. Vasilakos, "Cloud computing: Survey on energy efficiency," $A C M$ Computing Surveys (CSUR), vol. 47, no. 2, p. 33, 2014.

[3] R. N. Calheiros, R. Ranjan, A. Beloglazov, C. A. De Rose, and R. Buyya, "Cloudsim: a toolkit for modeling and simulation of cloud computing environments and evaluation of resource provisioning algorithms," Software: Practice and Experience, vol. 41, no. 1, pp. 23-50, 2011.

[4] T. Sturm, F. Jrad, and A. Streit, "Storage cloudsim - a simulation environment for cloud object storage infrastructures," in Proceedings of the 4th International Conference on Cloud Computing and Services Science ISBN 978-989-758-019-2, Barcelona, Spain. IEEE, April 2014, pp. 186-192.

[5] N. Grozev and R. Buyya, "Performance modelling and simulation of three-tier applications in cloud and multi-cloud environments," The Computer Journal, vol. 58, no. 1, pp. 1-22, 2015.

[6] S. Long and Y. Zhao, "A toolkit for modeling and simulating cloud data storage: An extension to cloudsim," in Control Engineering and Communication Technology (ICCECT), 2012 International Conference on. IEEE, 2012, pp. 597-600.

[7] B. Louis, "CloudSimDisk: Energy-Aware Storage Simulation in CloudSim." $\quad 2015$. [Online]. Available: http://pure.ltu.se/portal/en/studentthesis/ cloudsimdisk(232ab13a-1f2d-47f1-9960-4742eb1f95ff).html 